\title{
Determination of Shock Index Reference Values for Prepartum and Early Postpartum Period in Vaginal Delivery: A Prospective, Cross-sectional Study
}

\section{Vajinal Doğumda Prepartum ve Erken Postpartum Dönem İçin Şok İndeks Referans Değerlerinin Belirlenmesi: Prospektif, Kesitsel Çalışma}

\author{
$\underline{\text { Ihsan BAĞLI }}^{1}$ (D), İbrahim ANDAN ${ }^{2}$ (D) \\ ${ }^{1}$ University of Health Sciences Diyarbakir Gazi Yasargil Research and Training Hospital, Department of Obstetrics and \\ Gynecology, Diyarbakır, TURKEY \\ 2 Department of Anesthesiology and Reanimation, Dicle University Medical Faculty, Diyarbakır, TURKEY
}

Abstract

Background: The study aims to determine the variation of shock index (SI) before and after vaginal delivery and establish standard reference values in mothers who have not postpartum hemorrhage. Materials and Methods: A total of 2534 women who delivered vaginally were enrolled in the study. This prospective cross-sectional study was completed between November 2018 and September 2019 in our referral hospital. The exclusion criteria were anemia, cesarean delivery, maternal heart diseases, maternal thyroid disease, gestational hypertensive disorders, patients received epidural anesthesia, and less than a 34 week 0 day gestation, patients who had postpartum hemorrhage (PPH). We defined PPH as blood loss $>1000 \mathrm{ml}$ at the time of delivery. All patients' SI (heart rate/systolic blood pressure) were measured prepartum and at the 30th minutes, 1 st and 2 nd-hour postpartum period. Shock index reference ranges were measured separately according to $\mathrm{BMI}$, age, and parity groups.

Results: $10136 \mathrm{SI}$ values were assessed. The mean age \pm SD was $27.28 \pm 5.95$ years. The mean BMI \pm SD was $24.89 \pm 4.87 \mathrm{~kg} / \mathrm{m}^{2}$. The prepartum and postpartum 30 th minutes, $1 \mathrm{st}$ hour, and 2 nd hour mean shock index values were $0.76 \pm 0.07 ; 0.85 \pm 0.12 ; 0.84 \pm 0.12$ and $0.81 \pm 0.12$ respectively. The variation in SI values was significant by the Tukey test $(P<0.05)$.

Conclusions: For SI to be a diagnostic character in the issue of PPH, reference values must be known, so in this study shock index reference percentile ranges and mean \pm standard deviation of $\mathrm{SI}$ values were established.

Key Words: Pregnancy, Shock index, Vaginal delivery

öz.

Amaç: Vajinal doğumda doğum öncesi ve doğum sonrası erken dönemde postpartum kanaması tanısı almamış annelerde şok indeks referans değerlerinin belirlenmesi amaçlandı.

Materyal ve Metod: Çalışma prospektif kesitsel çalışma olarak tasarlandı. Kasım 2018 ve Eylül 2019 tarihleri arasında 2534 vaginal doğum yapmış hasta çalışmaya dahil edildi. Çalışma dışı bırakılma kriterleri olarak; anemi, sezaryen doğum, epidural anestezi uygulanmış hastalar, annede kalp hastalığı, gebeliğin hipertansif hastalıkları, annede tiroid hastalığı, 34 hafta altı doğumlar ve postpartum kanama tanısı almış olmak olarak belirlendi. Postpartum kanama tanısı olarak yaklaşı $1000 \mathrm{ml}$ üzerindeki kanama sınır değer olarak belirlendi. Şok indeks değerleri, kalp hızı/sistolik kan basıncı olarak hesaplandı. Hastaların doğum öncesi aktif travaydaki şok indeks değerleri ve doğum sonrası 30. dk, 1. saat ve 2. saat şok indeks değerleri not edildi. Şok indeks değerlerinin referans aralıkları hastaların vücut kitle indeksleri, yaşları ve önceki doğum sayılarına göre de ayrıca belirlendi.

Bulgular: 10136 şok indeks değeri hesaplandı. Hastaların ortalama \pm standart sapma yaşı $27.28 \pm 5.95$ olarak saptandı. Hastaların ortalama \pm standart sapma vücut kitle indeksi $24.89 \pm 4.87$ olarak bulundu. Doğum öncesi ve doğum sonrası 30 . $\mathrm{dk}, 1$. saat ve 2 . saat şok indeks ortalama \pm standart sapma değerleri sırasıyla $0.76 \pm 0.07 ; 0.85 \pm 0.12 ; 0.84 \pm 0.12$ ve $0.81 \pm 0.12$. Bu değerler arasındaki farklılık, Tukey testi kullanılarak istatistiksel olarak anlamlı bulundu $(\mathrm{p}<0.05)$.

Sonuç: Şok indeks değerlerinin postpartum kanama için tanı koydurucu bir belirteç olarak kullanılabilmesi için kanaması olmayan vajinal doğum yapmıs normal popülasyonda değerlendirilmesi ve referans aralıklarının belirlenmesi gerekmektedir. Çalışmamızda şok indeks referans değerlerinin yüzdelik değer aralıkları ve ortalamaları belirlenmiş ve tablolar halinde sunulmuştur.

Anahtar kelimeler: Gebelik, Şok index, Vajinal doğum
Corresponding Author/Sorumlu Yazar

Dr. İhsan BAĞLI

Diyarbakir Gazi Yasargil Egitim Arastirma Hastanesi Ek Bina, Urfa Yolu, Baglar, 21090 Diyarbakır/TURKEY

E-mail: ihsanbagli@gmail.com

Received / Geliş Tarihi: 25.03.2021

Accepted / Kabul Tarihi: 05.04.2021

DOI: $10.35440 /$ hutfd. 903404 


\section{Introduction}

The first description of shock index (SI), which is obtained by dividing the heart rate by the systolic blood pressure, was made in 1967 by Allgöver and Burri. Their study did not include pregnant individuals; their participants were diagnosed with gastrointestinal hemorrhage (1). Normal range of SI was stated as 0.5-0.7 for healthy adults in another study and in the another current study, for patients were not pregnant but were followed up for sepsis, the cut off value of the shock index for the initiation of inotropic agent was found to be $1 .(2,3)$. However, pregnancy induces substantial changes regarding the cardiovascular system. Therefore the shock index in pregnancy is different from a non-pregnant adult even during different periods of pregnancy (4). In pregnant women the blood volume increases by $4700-5200 \mathrm{~mL}$ and reaches the maximum value at $32 \mathrm{nd}$ week of pregnancy. Additionally, cardiac output rises by approximately $50 \%$ during pregnancy, increasing from 4.6 to $8.7 \mathrm{~L} / \mathrm{min}$ on average. The peak of cardia output occurs between 25 and 35 weeks and then remains stable until delivery $(5,6)$.

In obstetric populations, the SI was first used to identify the severity of blood loss in ectopic pregnancy (7). Postpartum hemorrhage (PPH) remains the leading cause of maternal death and morbidity worldwide (8). Most deaths due to hemorrhage happens at the postpartum period in both highincome (49.1\%) and low/middle-income countries (73\%) (9).

In the face of this serious vital problem, sometimes health care workers can be delayed at first intervention and take action only when the bleeding becomes significant. Health workers often have difficulty describing PPH to each other. Confirmation of this prediction sometimes requires a laboratory test before initiating treatment. Moreover there is still controversy between different organisations. The World Health Organization (WHO) describes PPH for vaginal delivery as above $500 \mathrm{ml}$ blood loss and for a cesarean section as above $1000 \mathrm{ml}$ blood loss, whereas the American College of Obstetricians and Gynecologists (ACOG) defined $\mathrm{PPH}$ as blood loss $>1000 \mathrm{ml}$ at the time of delivery, independent of the mode of delivery in their recent practice bulletin number $183(10,11)$. This bleeding above $1000 \mathrm{ml}$ corresponds to decreasing $10 \%$ in hematocrit and it has been proposed as an alternative marker to define significant hemorrhage. However, the changing hematocrit concentrations are often delayed to respond hemorrhage and may not reflect current hematologic status, and may not be useful in the setting with acute haemorrhage (11).

Instead of the WHO's recommendation about the definition of PPH as blood loss above $500 \mathrm{ml}$ in vaginal delivery, and low accuracy of visual estimation of blood loss for PPH $(10,12)$, an article published in the year of 2018 at the American Journal of Obstetrics and Gynecology emphasized that shock index can be more useful for defining $\mathrm{PPH}$ (13). According to many studies which accepted PPH as blood loss above $500 \mathrm{ml}$ in vaginal delivery, SI greater than 0.9 confirmed the diagnosis of postpartum hemorrhage requiring transfusion (14-16). No study discusses SI values according to ACOG's new PPH definition as blood loss > $1000 \mathrm{ml}$ at the time of delivery, regardless of the mode of delivery. Hence we aimed to define the normal standard ranges of SI values at prepartum and postpartum 30th minutes, 1 st hour, and 2 nd hour in vaginal delivery when $\mathrm{PPH}$ was accepted as blood loss above $1000 \mathrm{ml}$.

\section{Materials and Methods}

This is a prospective, cross-sectional study. Data were collected between December 2018 and June 2019 at our tertiary referral center. The study was approved by the local ethics committee (Health Sciences University Gazi Yaşargil Training and Research Hospital clinical research ethics committee Date: 26/10/2018 protocol number: 166). In that time interval, 10955 deliveries occurred. Active management protocol was applied to each patient for the third stage of labor 15 . We collected our data during 24-hour delivery room shift. Over that the study period, we managed the labor process of 3541 patients, 477 of them were excluded from the study because they underwent cesarean section.

Data of 3064 pregnant women who delivered vaginally without epidural anesthesia were collected. Postpartum hemorrhage was excluded by visual estimation of blood loss, and this method was confirmed with less than $10 \%$ decrease in hematocrit level, which was routinely monitored with complete blood count test at six hours after delivery. A decrease of more than 10 percent in prepartum and postpartum hematocrit indicates an average of over $1000 \mathrm{ml}$ of bleeding. So that, we used the recommendation of ACOG for the definition of PPH as $>1000 \mathrm{ml}$ blood loss at the time of vaginal delivery (11). So, 180 patients with PPH who had more than $10 \%$ decrease in hematocrit level or had blood loss accompanied by signs or symptoms of hypovolemia despite less than $10 \%$ decreased hematocrit levels were excluded.

156 patients with chronic hypertension, 62 patients with transfused prepartum due to anemia, 36 instrumental delivery with vacuum, 96 patients with preeclampsia were excluded from the remaining 2884 women; therefore, 2534 patients were enrolled. With the birth of the fetus, 2534 patients were started with $1000 \mathrm{cc}$ of $0.9 \% \mathrm{NaCl}$ with $20 \mathrm{U}$ of oxytocin intravenously, set to $150 \mathrm{ml}$ per hour according to ACOG recommendation (17).

The demographic characteristics of all patients, including age, parity, and body mass index

(BMI), were recorded. Heart rate, and systolic and diastolic blood pressure were taken when the pregnants were admitted to the labor room (prepartum), and all measures were taken again at 30th minutes, 1 hour, and 2 hours after delivery, with an automated monitor.

Age groups were divided as $\leq 19$ (n:218), 20-24 (n:696), 25- 
29 (n:748), 30-35 (n: 496) and $\geq 35$ (n: 340). BMI groups were divided as 18 ( $n: 248), 19-24$ ( $\mathrm{n:1098),} \mathrm{25-29} \mathrm{(n:762),}$ and 30-34 (n:426). Parity groups were divided as nulliparity ( $\mathrm{n:760)}$, multiparity (delivery number 1-4) (n:1538), and grand-multiparity (delivery number $\geq 5)(n: 236)$.

Statistical analysis was made using the NCSS (Number Cruncher Statistical System) 2007 Statistical Software Program. Descriptive statistical methods (mean, standard deviation, median, and interquartile range), as well as the unidirectional variance analysis, were used when the variables showed normal distribution tested by Shapiro - Wilk normality test. To identify variation among the prepartum and postpartum periods, the mean of SI values observed for the following intervals were used in the analysis: prepartum and postpartum 30th min, 1st hour, 2nd hour. The differences in SI values were evaluated by analysis of the Tukey test for independent measurements ( $p<0.05$ was accepted significant). According to age, BMI, and parity, the 5th, 10th, 25th, 50th, 75th, 90th, 95th percentiles of SI values were calculated for each group.

\section{Results}

A total of 2534 women were included, and 10136 values of SI were processed. The mean patient's age \pm SD was $27.28 \pm 5.95$ years. The mean BMI \pm SD was $24.89 \pm 4.87 \mathrm{~kg} / \mathrm{m} 2$. The prepartum, and 30th min, 1st and 2 nd hour postpartum mean SI values were $0.76 \pm$ $0.07 ; 0.85 \pm 0.12 ; 0.84 \pm 0.12$ and $0.81 \pm 0.12$ respectively. There were significant differences between prepartum and postpartum SI values $(p<0.05)$. Postpartum 30 min and 1st hour mean SI values were significantly higher than the other SI values $(p<0.05)$. These findings are presented in Table 1.

Table 1.Patients' Descriptive Characteristics

\begin{tabular}{lcccc}
\hline N (2534) & Mean \pm SD & Median (IQR) & Min. & Max. \\
\hline Age (year) & $27.28 \pm 5.95$ & $26(23-32)$ & 16 & 46 \\
Gravidity & $2.79 \pm 1.70$ & $3(1-4)$ & 1 & 13 \\
Parity & $2.32 \pm 1.65$ & $2(1-3)$ & 0 & 12 \\
BMI (kg/m ${ }^{2}$ ) & $24.89 \pm 4.87$ & $24(21-28)$ & 17 & 38 \\
Prepartum SI & $0.76 \pm 0.07^{\text {a }}$ & $0.76(0.72-0.8)$ & 0.49 & 1,02 \\
Pospartum 30th min. SI & $0.85 \pm 0.12^{\text {a,b }}$ & $0.86(0.78-0.91)$ & 0.44 & 1.03 \\
Postpartum 1th hour SI & $0.84 \pm 0.12^{\text {a,b }}$ & $0.84(0.78-0.86)$ & 0.43 & 1.04 \\
Postpartum 2nd hour SI & $0.81 \pm 0.12^{\text {a }}$ & $0.81(0.69-0.84)$ & 0.41 & 1.01 \\
\hline
\end{tabular}

${ }^{a}$ There were significant differences between prepartum and postpartum SI (Shock Index) values by Tukey test ( $p<0.05$ )

${ }^{b}$ Postpartum 30 min and 1st hour mean SI values were significantly higher than the other SI values by Tukey test $(p<0.05)$

There were 532 (21\% of total birth) patients with episiotomy. A total of 760 patients (30\% of total birth) were primiparous. Table 2 shows the percentiles of SI stratified by maternal age group.

Table 3 shows the percentiles of SI stratified by parity group, and Table 4 illustrates the percentiles of $\mathrm{SI}$ among patients in the BMI group. There were significant differences between $\leq 19$ age group and others.
Table 2. Reference values of shock index (SI) according to age groups

\begin{tabular}{|c|c|c|c|c|c|c|c|c|}
\hline & \multirow{2}{*}{$\begin{array}{l}\text { Age } \\
\text { (year) }\end{array}$} & \multicolumn{7}{|c|}{ Percentiles } \\
\hline & & $5 \mathrm{P}$ & $10 \mathrm{P}$ & $25 \mathrm{P}$ & $50 \mathrm{P}$ & $75 \mathrm{P}$ & $90 \mathrm{P}$ & $95 \mathrm{P}$ \\
\hline \multirow[t]{5}{*}{ Prepartum SI } & $<19$ & 0,632 & 0,655 & 0,709 & 0,740 & 0,800 & 0,820 & 0,861 \\
\hline & $20-24$ & 0,636 & \begin{tabular}{|l|}
0,667 \\
\end{tabular} & 0,709 & 0,760 & 0,800 & 0,840 & 0,860 \\
\hline & $25-29$ & 0,655 & 0,667 & 0,717 & 0,760 & 0,800 & 0,840 & 0,860 \\
\hline & $30-35$ & 0,615 & 0,667 & 0,720 & 0,764 & 0,800 & 0,844 & 0,881 \\
\hline & $>35$ & 0,633 & 0,667 & 0,709 & 0,762 & 0,800 & 0,844 & 0,860 \\
\hline \multirow{5}{*}{$\begin{array}{l}\text { Postpartum } \\
\text { 30th min. SI }\end{array}$} & $<19$ & 0,661 & 0,691 & 0,776 & 0,860 & 0,925 & 1,020 & 1,082 \\
\hline & $20-24$ & 0,691 & 0,709 & 0,778 & 0,855 & 0,900 & 1,000 & 1,040 \\
\hline & $25-29$ & 0,691 & 0,709 & 0,782 & 0,860 & 0,918 & 0,980 & 1,020 \\
\hline & $30-35$ & 0,664 & 0,700 & 0,782 & 0,860 & 0,907 & 1,000 & 1,043 \\
\hline & $>35$ & 0,683 & 0,720 & 0,782 & 0,860 & 0,900 & 0,980 & 1,020 \\
\hline \multirow{5}{*}{$\begin{array}{l}\text { Postpartum } \\
\text { 1th hour SI }\end{array}$} & $<19$ & 0,718 & 0,727 & 0,781 & 0,860 & 0,920 & 1,044 & 1,139 \\
\hline & $20-24$ & 0,673 & 0,720 & 0,764 & 0,840 & 0,909 & 0,980 & 1,025 \\
\hline & $25-29$ & 0,690 & 0,727 & 0,780 & 0,847 & 0,920 & 0,980 & 1,022 \\
\hline & $30-35$ & 0,670 & 0,709 & 0,764 & 0,840 & 0,920 & 0,980 & 1,042 \\
\hline & $>35$ & 0,691 & 0,720 & 0,782 & 0,840 & 0,891 & 0,980 & 1,020 \\
\hline \multirow{5}{*}{$\begin{array}{l}\text { Postpartum } \\
\text { 2nd hour SI }\end{array}$} & $<19$ & 0,699 & 0,750 & 0,791 & 0,860 & 0,956 & 1,110 & 1,045 \\
\hline & $20-24$ & 0,727 & 0,760 & 0,800 & 0,860 & 0,956 & 1,050 & 1,111 \\
\hline & $25-29$ & 0,727 & 0,764 & 0,800 & 0,860 & 0,933 & 1,030 & 1,089 \\
\hline & $30-35$ & 0,700 & 0,740 & 0,782 & 0,860 & 0,926 & 1,020 & 1,089 \\
\hline & $>35$ & 0,727 & 0,764 & 0,791 & 0,847 & 0,927 & 1,000 & 1,089 \\
\hline
\end{tabular}

Table 3. Reference values of shock index (SI) according to parity groups

\begin{tabular}{|c|c|c|c|c|c|c|c|c|}
\hline & & \multicolumn{7}{|c|}{ Percentiles } \\
\hline & Parity & $5 \mathrm{P}$ & $10 \mathrm{P}$ & $25 \mathrm{P}$ & $50 \mathrm{P}$ & $75 \mathrm{P}$ & $90 \mathrm{P}$ & $95 \mathrm{P}$ \\
\hline \multirow[t]{3}{*}{ Prepartum SI } & $\begin{array}{l}\text { Nullipa- } \\
\text { raous }\end{array}$ & 0,631 & 0,665 & 0,709 & 0,760 & 0,800 & 0,840 & 0,867 \\
\hline & $\begin{array}{l}\text { 1-4 Multi- } \\
\text { paraous }\end{array}$ & 0,636 & 0,667 & 0,717 & 0,760 & 0,800 & 0,840 & 0,860 \\
\hline & $\begin{array}{l}>5 \text { Grand } \\
\text { Multipa- } \\
\text { raous }\end{array}$ & 0,631 & 0,655 & 0,683 & 0,753 & 0,800 & 0,840 & 0,889 \\
\hline \multirow[t]{3}{*}{$\begin{array}{l}\text { Pospartum } \\
\text { 30th } \min \mathrm{SI}\end{array}$} & $\begin{array}{l}\text { Nullipa- } \\
\text { raous }\end{array}$ & 0,691 & 0,709 & 0,782 & 0,860 & 0,933 & 1,040 & 1,100 \\
\hline & $\begin{array}{l}\text { 1-4 Multi- } \\
\text { paraous }\end{array}$ & 0,686 & 0,709 & 0,782 & 0,860 & 0,909 & 0,980 & 1,020 \\
\hline & $\begin{array}{l}>5 \text { Grand } \\
\text { Multipa- } \\
\text { raous }\end{array}$ & 0,629 & 0,700 & 0,764 & 0,835 & 0,882 & 1,000 & 1,020 \\
\hline \multirow[t]{3}{*}{$\begin{array}{l}\text { Postpartum } \\
\text { 1st hour SI }\end{array}$} & $\begin{array}{l}\text { Nullipa- } \\
\text { raous }\end{array}$ & 0,720 & 0,730 & 0,800 & 0,870 & 0,920 & 1,002 & 1,080 \\
\hline & $\begin{array}{l}\text { 1-4 Multi- } \\
\text { par }\end{array}$ & 0,673 & 0,720 & 0,767 & 0,840 & 0,920 & 0,980 & 1,040 \\
\hline & $\begin{array}{l}>5 \text { Grand } \\
\text { Multipar }\end{array}$ & 0,662 & 0,717 & 0,764 & 0,828 & 0,900 & 0,980 & 1,020 \\
\hline \multirow[t]{3}{*}{$\begin{array}{l}\text { Postpartum } \\
\text { 2nd hour SI }\end{array}$} & $\begin{array}{l}\text { Nullipa- } \\
\text { raous }\end{array}$ & 0,727 & 0,766 & 0,800 & 0,860 & 0,927 & 1,059 & 1,020 \\
\hline & $\begin{array}{l}\text { 1-4 Multi- } \\
\text { paraous }\end{array}$ & 0,727 & 0,760 & 0,800 & 0,860 & 0,950 & 1,030 & 1,091 \\
\hline & $\begin{array}{l}>5 \text { Grand } \\
\text { Multipa- } \\
\text { raous }\end{array}$ & 0,673 & 0,727 & 0,782 & 0,842 & 0,927 & 1,030 & 1,011 \\
\hline
\end{tabular}

$\leq 19$ age group had higher SI values at postpartum 1st and 2 nd hour ( $p$ : 0.009) and detailed values were shown in table 5 . There were significant differences between nulliparity and multiparity for postpartum 30. min. (p: 0.002) and postpartum 1st hour (p: 0.001) respectively. Nulliparity had higher SI values for mentioned times and detailed information stated in table 6.

There were no significant differences between BMI groups for SI values (p: 0.083 for prepartum SI, p: 0.136 for postpartum 30. $\mathrm{min} \mathrm{SI,} \mathrm{p:} 0.623$ for postpartum 1st hour, p: 0.893 for postpartum 2 nd hour), detailed values were shown at table 7 . 
Table 4. Reference percentile values of shock index (SI) according to BMI groups

\begin{tabular}{|c|c|c|c|c|c|c|c|c|}
\hline & \multicolumn{8}{|c|}{ Percentiles } \\
\hline & $\begin{array}{l}\mathrm{BMI} \\
\left(\mathrm{kg} / \mathrm{m}^{2}\right)\end{array}$ & $5 P$ & $10 \mathrm{P}$ & $25 \mathrm{P}$ & $50 \mathrm{P}$ & $75 P$ & $90 \mathrm{P}$ & $95 \mathrm{P}$ \\
\hline \multirow{4}{*}{ Prepartum SI } & $<18$ & 0,655 & 0,670 & \begin{tabular}{|l|l|}
0,727 \\
\end{tabular} & 0,780 & 0,800 & 0,840 & 0,860 \\
\hline & $19-24$ & 0,636 & 0,667 & 0,719 & 0,760 & 0,800 & 0,844 & 0,880 \\
\hline & $25-29$ & 0,633 & 0,667 & 0,709 & 0,760 & 0,800 & 0,820 & 0,860 \\
\hline & $30-34$ & 0,634 & 0,667 & \begin{tabular}{|l|}
0,709 \\
\end{tabular} & 0,760 & 0,800 & 0,840 & 0,860 \\
\hline \multirow{4}{*}{$\begin{array}{l}\text { Pospartum } \\
\text { 30th min. SI }\end{array}$} & $<18$ & 0,673 & 0,728 & 0,800 & 0,873 & 0,940 & 1,018 & 1,060 \\
\hline & 19-24 & 0,683 & 0,709 & 0,782 & 0,860 & 0,909 & 0,980 & 1,020 \\
\hline & $25-29$ & 0,683 & 0,709 & 0,780 & 0,860 & 0,900 & 1,000 & 1,060 \\
\hline & $30-34$ & 0,691 & 0,709 & 0,780 & 0,860 & 0,900 & 0,980 & 1,060 \\
\hline \multirow{4}{*}{$\begin{array}{l}\text { Postpartum } \\
\text { 1th hour SI }\end{array}$} & $<18 \mathrm{~B}$ & 0,671 & 0,709 & 0,782 & 0,860 & 0,920 & 1,000 & 1,055 \\
\hline & $19-24$ & 0,691 & $\begin{array}{ll}0,727 \\
\end{array}$ & 0,780 & 0,840 & 0,920 & 0,980 & 1,020 \\
\hline & 25-29 & 0,670 & 0,720 & 0,764 & 0,840 & 0,920 & 0,980 & 1,054 \\
\hline & $30-34$ & 0,694 & 0,733 & 0,764 & 0,840 & 0,911 & 0,980 & 1,040 \\
\hline \multirow{4}{*}{$\begin{array}{l}\text { Postpartum } \\
\text { 2nd hour SI }\end{array}$} & $<18$ & 0,727 & 0,759 & 0,782 & 0,860 & 0,954 & 1,030 & 1,111 \\
\hline & 19-24 & 0,727 & 0,764 & 0,800 & 0,860 & 0,940 & 1,023 & 1,090 \\
\hline & $25-29$ & 0,700 & 0,744 & 0,782 & 0,860 & 0,940 & 1,056 & 1,011 \\
\hline & $30-34$ & 0,700 & 0,764 & 0,800 & 0,860 & 0,933 & 1,025 & 1,089 \\
\hline
\end{tabular}

Table 5. Shock index values between age groups

\begin{tabular}{|c|c|c|c|c|c|c|}
\hline & Age & $\mathrm{N}$ & Mean $\pm S D$ & Median (IQR) & Min & Max \\
\hline \multirow{6}{*}{ Prepartum SI } & $<19$ & 218 & $0,749 \pm 0,079$ & $\begin{array}{c}0,740 \\
(0,710-0,800)\end{array}$ & 0,49 & 1,02 \\
\hline & $20-24$ & 696 & $0,756 \pm 0,068$ & $\begin{array}{c}0,760 \\
(0,710-0,800)\end{array}$ & 0,51 & 1,02 \\
\hline & $25-29$ & 784 & $0,757 \pm 0,067$ & $\begin{array}{c}0,760 \\
(0,720-0,800)\end{array}$ & 0,49 & 0,91 \\
\hline & $30-35$ & 496 & $0,758 \pm 0,083$ & $\begin{array}{c}0,764 \\
(0,720-0,800)\end{array}$ & 0,49 & 1,02 \\
\hline & $>35$ & 340 & $0,757 \pm 0,076$ & $\begin{array}{c}0,762 \\
(0,710-0,800)\end{array}$ & 0,49 & 1,02 \\
\hline & \multicolumn{6}{|c|}{$\mathbf{p}^{*} 0,643$} \\
\hline \multirow{6}{*}{ Pospartum 30.min SI } & $<19$ & 218 & $0,859 \pm 0,144$ & $\begin{array}{c}0,760 \\
(0,780-0,930)\end{array}$ & 0,44 & 1,14 \\
\hline & $20-24$ & 696 & $0,851 \pm 0,117$ & $\begin{array}{c}0,860 \\
(0,780-0,900)\end{array}$ & 0,55 & 1,10 \\
\hline & $25-29$ & 784 & $0,851 \pm 0,111$ & $\begin{array}{c}0,855 \\
(0,780-0,920)\end{array}$ & 0,44 & 1,04 \\
\hline & $30-35$ & 496 & $0,848 \pm 0,121$ & $\begin{array}{c}0,860 \\
(0,780-0,910)\end{array}$ & 0,44 & 1,02 \\
\hline & & 340 & $0,846 \pm 0,110$ & $\begin{array}{c}0,860 \\
(0,780-0,900)\end{array}$ & 0,44 & 1,02 \\
\hline & \multicolumn{6}{|c|}{$\mathbf{p}^{*} 0,765$} \\
\hline \multirow{6}{*}{ Postpartum 1th hour SI } & $<19$ & 218 & $0,874 \pm 0,145$ & $\begin{array}{c}0,860 \\
(0,780-0,920) \\
\end{array}$ & 0,43 & 1,13 \\
\hline & $20-24$ & 696 & $0,847 \pm 0,111$ & $\begin{array}{c}0,860 \\
(0,760-0,910)\end{array}$ & 0,60 & 1,08 \\
\hline & $25-29$ & 784 & $0,854 \pm 0,113$ & $\begin{array}{c}0,860 \\
(0,780-0,920)\end{array}$ & 0,43 & 1,03 \\
\hline & $30-35$ & 496 & $0,846 \pm 0,121$ & $\begin{array}{c}0,840 \\
(0,760-0,920)\end{array}$ & 0,43 & 1,14 \\
\hline & & 340 & $0,842 \pm 0,100$ & $\begin{array}{c}0,847 \\
(0,780-0,890)\end{array}$ & 0,43 & 1,09 \\
\hline & \multicolumn{6}{|c|}{$p^{*} 0,009$} \\
\hline \multirow{6}{*}{ Postpartum 2nd hour SI } & $<19$ & 218 & $0,892 \pm 0,139$ & $\begin{array}{c}0,840 \\
(0,790-0,960)\end{array}$ & 0,64 & 1,16 \\
\hline & $20-24$ & 696 & $0,883 \pm 0,124$ & $\begin{array}{c}0,840 \\
(0,800-0,960)\end{array}$ & 0,55 & 1,08 \\
\hline & $25-29$ & 784 & $0,878 \pm 0,115$ & $\begin{array}{c}0,840 \\
(0,800-0,930)\end{array}$ & 0,62 & 1,06 \\
\hline & $30-35$ & 496 & $0,867 \pm 0,115$ & $\begin{array}{c}0,860 \\
(0,780-0,930)\end{array}$ & 0,64 & 1,08 \\
\hline & $>35$ & 340 & $0,866 \pm 0,107$ & $\begin{array}{c}0,860 \\
(0,790-0,930)\end{array}$ & 0,62 & 1,01 \\
\hline & \multicolumn{6}{|c|}{$\begin{array}{l}p^{*} 0,018 \\
\end{array}$} \\
\hline
\end{tabular}

* Unidirectional Variance Analysis
Table 6. Shock index values between parity groups

\begin{tabular}{|c|c|c|c|c|c|c|}
\hline & & $\mathbf{N}$ & Mean \pm SD & Median (IQR) & Min. & Max. \\
\hline \multirow{4}{*}{ Prepartum SI } & Nullipar & 760 & $0,75 \pm 0,073$ & $\begin{array}{c}0,760 \\
(0,709-0,800)\end{array}$ & 0,540 & 0,910 \\
\hline & $\begin{array}{l}\text { 1-4 Multi- } \\
\text { par }\end{array}$ & 1538 & $0,758 \pm 0,071$ & $\begin{array}{c}0,760 \\
(0,717-0,800) \\
\end{array}$ & 0,490 & 1,020 \\
\hline & $\begin{array}{l}>5 \text { Grand } \\
\text { Multipar }\end{array}$ & 236 & $0,752 \pm 0,085$ & $\begin{array}{c}0,753 \\
(0,683-0,800)\end{array}$ & 0,490 & 1,020 \\
\hline & $\mathbf{p}^{*} 0,138$ & & & & & \\
\hline \multirow{4}{*}{$\begin{array}{l}\text { Pospartum } \\
\text { 30.min SI }\end{array}$} & Nullipar & 760 & $0,871 \pm 0,138$ & $\begin{array}{c}0,860 \\
(0,782-0,933) \\
\end{array}$ & 0,640 & 1,02 \\
\hline & $\begin{array}{l}\text { 1-4 Multi- } \\
\text { par }\end{array}$ & 1538 & $0,849 \pm 0,112$ & $\begin{array}{c}0,860 \\
(0,782-0,909) \\
\end{array}$ & 0,440 & 1,02 \\
\hline & $\begin{array}{l}>5 \text { Grand } \\
\text { Multipar }\end{array}$ & 236 & $0,839 \pm 0,131$ & $\begin{array}{c}0,835 \\
(0,764-0,882)\end{array}$ & 0,440 & 0,91 \\
\hline & $p^{*} 0,002$ & & & & & \\
\hline \multirow{4}{*}{$\begin{array}{l}\text { Postpartum } \\
\text { 1th hour SI }\end{array}$} & Nullipar & 760 & $0,873 \pm 0,125$ & $\begin{array}{c}0,870 \\
(0,800-0,920) \\
\end{array}$ & 0,600 & 1,02 \\
\hline & $\begin{array}{l}\text { 1-4 Multi- } \\
\text { par }\end{array}$ & 1538 & $0,848 \pm 0,113$ & $\begin{array}{c}0,840 \\
(0,767-0,920) \\
\end{array}$ & 0,430 & \\
\hline & $\begin{array}{l}>5 \text { Grand } \\
\text { Multipar }\end{array}$ & 236 & $0,841 \pm 0,121$ & $\begin{array}{c}0,828 \\
(0,764-0,900) \\
\end{array}$ & 0,430 & 1,14 \\
\hline & $p^{*} 0,001$ & & & & & \\
\hline \multirow{4}{*}{$\begin{array}{l}\text { Postpartum } \\
\text { 2nd hour SI }\end{array}$} & Nullipar & 760 & $0,881 \pm 0,123$ & $\begin{array}{c}0,860 \\
(0,800-0,927)\end{array}$ & 0,640 & 1,04 \\
\hline & $\begin{array}{ll}1-4 & \text { Multi- } \\
\text { par }\end{array}$ & 1538 & $0,878 \pm 0,117$ & $\begin{array}{c}0,860 \\
(0,800-0,950) \\
\end{array}$ & 0,550 & 1,02 \\
\hline & $\begin{array}{l}>5 \text { Grand } \\
\text { Multipar }\end{array}$ & 236 & $0,863 \pm 0,127$ & $\begin{array}{c}0,842 \\
(0,782-0,927)\end{array}$ & 0,620 & 1,02 \\
\hline & $p^{*} 0,152$ & & & & & \\
\hline
\end{tabular}

*Unidirectional Variance Analysis

Table 7. Shock index values between BMI groups

\begin{tabular}{|c|c|c|c|c|c|c|}
\hline & BMI & $\mathrm{N}$ & Mean $\pm S D$ & Median (IQR) & Min. & Max. \\
\hline \multirow{5}{*}{ Prepartum SI } & $<18 \mathrm{BMI}$ & 248 & $0,763 \pm 0,072$ & $\begin{array}{c}0,780 \\
(0,727-0,800)\end{array}$ & 0,490 & 0,930 \\
\hline & 19-24 BMI & 1098 & $0,759 \pm 0,073$ & $\begin{array}{c}0,760 \\
(0,719-0,800)\end{array}$ & 0,490 & 1,020 \\
\hline & 25-29 BMI & 762 & $0,751 \pm 0,074$ & $\begin{array}{c}0,760 \\
(0,709-0,800)\end{array}$ & 0,490 & 1,020 \\
\hline & 30-34 BMI & 426 & $0,755 \pm 0,072$ & $\begin{array}{c}0,760 \\
(0,709-0,800)\end{array}$ & 0,490 & 1,020 \\
\hline & \multicolumn{6}{|l|}{$\mathbf{p}^{*} 0,083$} \\
\hline \multirow{5}{*}{$\begin{array}{l}\text { Pospartum } \\
\text { 30. } \min \mathrm{SI}\end{array}$} & $<18 \mathrm{BMI}$ & 248 & $0,867 \pm 0,118$ & $\begin{array}{c}0,873 \\
(0,800-0,940)\end{array}$ & 0,440 & 1,02 \\
\hline & 19-24 BMI & 1098 & $0,848 \pm 0,114$ & $\begin{array}{c}0,860 \\
(0,782-0,909)\end{array}$ & 0,440 & 1,02 \\
\hline & 25-29 BMI & 762 & $0,849 \pm 0,123$ & $\begin{array}{c}0,860 \\
(0,780-0,900)\end{array}$ & 0,440 & 0,91 \\
\hline & 30-34 BMI & 426 & $0,85 \pm 0,118$ & $\begin{array}{c}0,860 \\
(0,780-0,900)\end{array}$ & 0,440 & 1,02 \\
\hline & \multicolumn{4}{|l|}{$\mathbf{p}^{*} 0,136$} & & \\
\hline \multirow{5}{*}{$\begin{array}{l}\text { Postpartum } \\
\text { 1th hour SI }\end{array}$} & $<18 \mathrm{BMI}$ & 248 & $0,86 \pm 0,114$ & $\begin{array}{c}0,860 \\
(0,782-0,920)\end{array}$ & 0,430 & \\
\hline & 19-24 BMI & 1098 & $0,85 \pm 0,111$ & $\begin{array}{c}0,840(0,780- \\
0,920)\end{array}$ & 0,430 & 1,14 \\
\hline & 25-29 BMI & 762 & $0,849 \pm 0,126$ & $\begin{array}{c}0,840 \\
(0,764-0,920)\end{array}$ & 0,430 & 1,10 \\
\hline & 30-34 BMI & 426 & $0,85 \pm 0,112$ & $\begin{array}{c}0,840 \\
(0,764-0,911)\end{array}$ & 0,430 & 1,04 \\
\hline & \multicolumn{6}{|l|}{$\mathbf{p}^{*} 0,623$} \\
\hline \multirow{5}{*}{$\begin{array}{l}\text { Postpartum } \\
\text { 2nd hour SI }\end{array}$} & $<18 \mathrm{BMI}$ & 248 & $0,88 \pm 0,118$ & $\begin{array}{c}0,860 \\
(0,782-0,954)\end{array}$ & 0,620 & 1,02 \\
\hline & 19-24 BMI & 1098 & $0,878 \pm 0,116$ & $\begin{array}{c}0,860 \\
(0,800-0,940)\end{array}$ & 0,550 & 1,02 \\
\hline & 25-29 BMI & 762 & $0,875 \pm 0,128$ & $\begin{array}{c}0,860 \\
(0,782-0,94)\end{array}$ & 0,550 & 1,02 \\
\hline & 30-34 BMI & 426 & $0,874 \pm 0,11$ & $\begin{array}{c}0,860 \\
(0,800-0,933)\end{array}$ & 0,640 & 0,91 \\
\hline & \multicolumn{6}{|l|}{$\mathbf{p}^{*} 0,893$} \\
\hline
\end{tabular}

* Unidirectional Variance Analysis

\section{Discussion}

There are some parameters and methods for evaluating blood loss after delivery, such as using a calibrated drape placed under the patient's buttocks, a decreased hematocrit level over $10 \%$, or a visual estimation of blood loss (10, 18). Unfortunately, obstetricians sometimes suffer from these subjective methods and late laboratory results. 
There is a new insight tool for SI that is being recommended as a possible indicator of PPH (13). Heart rate and systolic or diastolic blood pressure alone is not enough for the evaluation of postpartum blood loss $(19,20)$. In this study, we aimed to demonstrate the reference values of $\mathrm{SI}$ both prepartum and immediately postpartum.

The mean SI was still higher after postpartum at the second hour $(0.81 \pm 0.12)$ compared to prepartum $(0.76 \pm 0.07)$. In a previous study, for gestational ages between 33 week 0 day -36 week 6 days and above 37 weeks 0 day, the mean \pm SD SI values found were $0.82 \pm 0.14$ and $0.79 \pm 0.13$ respectively (21). These values are slightly higher than in the present study which found the prepartum SI as $0.76 \pm 0.07$. In non-obstetrics, SI ranges were reported as 0.5-0.7 9, even in the prepartum period in our study the SI was higher $(0.76 \pm 0.07)$. In a large study, Taylor et al. found that the SI range was 0.46 ( 5 th percentile) -1.07 (95th percentile) (22), and in another study, the mean SI values were 0.70; 0.69; 0.68 at 30 minutes, one hour, and 2 hours after delivery respectively (23). However, our study's mean SI values were $0.85 ; 0.84 ; 0.81$ at 30 minutes, one hour, and 2 hours postpartum respectively. We can explain why our values were higher than of those because while our study depended on the definition of PPH as blood loss $>1000 \mathrm{ml}$ at delivery according to ACOG 2017, Taylor et al.'s definition method of PPH was unclear and Borovac et al. defined PPH as blood loss $>500 \mathrm{ml}$ in vaginal delivery according to the WHO recommendation $(9,10,22,23)$. In 2019, Borovac et al. observed that SI tended to stabilize minutes after birth, in our study this stabilization occurred at 30 minutes after birth (23).

Also, we calculated the percentiles of SI between the BMI, parity, and age groups. A small woman may become symptomatic with 300-500 cc of bleeding after birth, while a large woman may not give symptoms or signs with $1000 \mathrm{cc}$ of bleeding (13). First delivery can be scary for young women and this led us to think about encountering a high heart rate. For these reasons, we decided to create the BMI, parity, and age groups. We did not find significant differences between the BMI groups but nulliparity and youngest women had high SI values at some postpartum period and these were stated at the result section. Borovac et al. studied postpartum SI values between BMI and age groups and they did not find any differences for age groups but their study population was very small as 186 participants while ours had 2534 participants (23).

Cardiac output (CO) is a multiplier of systolic blood pressure, and $\mathrm{CO}$ is increased in pregnancy. Following delivery there is an immediate rise in CO (increases by $60-80 \%$ ), then, a rapid decline occurs to pre-labor values approximately within one hour of delivery (24). Cardiac physiology changes at the different stages of labor, so SI could be affected by these changes. To the best of our knowledge, there was no other study that measured prepartum and postpartum SI at an exact time in a large cohort in the literature. In our study, we demonstrate the changes of SI in both the prepartum and especially the early postpartum period.

In a current study, a group with a BMI of $30 \mathrm{~kg} / \mathrm{m} 2$ or more had the lowest mean SI values compared with the 19-24 $\mathrm{kg} / \mathrm{m} 2$ and $25-29 \mathrm{~kg} / \mathrm{m} 2$ groups; however, in our groups there was no difference in SI values regarding BMI (23).

Patients with episiotomy or perineal tears are likely to have more pain and the result of pain an increased heart rate is inevitable (25). This is a weakness of our study because we did not differentiate patients with or without an episiotomy/perineal tears. First pregnancies in our region are often referred to as our hospital, and episiotomy is generally performed in the first births. Because the pain of an episiotomy or small perineal tears increase heart rate, our SI reference values were higher than previous studies for the postpartum period $(22,23)$. Our episiotomy rate was $21 \%$ while other studies' episiotomy rate was $31 \% .21 \mathrm{In}$ this study, we only made a visual estimation of diagnoses of PPH which confirmation via not decline over $10 \%$ in hematocrit levels $(11,12)$, because we did not have a calibrated drape for measure blood loss, nor did we evaluate weight gauzes and compresses for any patient. Similarly, Taylor et al. and Nathan et al. also used the method of visual estimation of blood loss during the postpartum period, differently, they defined PPH as blood loss over $500 \mathrm{ml}$ after vaginal delivery $(22,26)$, Borovac et al. used special bedspread that measures the blood loss (23). On the other hand according to many studies, the shock index threshold of $>0.9$ is indicating a need for referral, $>1.4$ indicating the urgent need for intervention in tertiary facilities and $>1.7$ indicating a high chance of adverse outcome (14-15), and in another study, an obstetric SI of more than 1 seems to be a useful adjunct in predicting the need for blood products (16). However, our postpartum 30th min and 2ndhour SI mean values were 0.85 and 0.84 respectively, these are very near to 0.9 and our patients had no even diagnoses of PPH. Tanacan et al. defined SI and peak SI at postpartum 1th hour as $0.76 \pm 0.13$ and $0.82 \pm 0.09$ respectively in healty control group and they used visual estimation of blood loss method for PPH ( $>1000 \mathrm{ml}$ at $\mathrm{CS}$ and $>500 \mathrm{ml}$ at vaginal delivery) (27). The reason for these differences can be explained since using a different definition of postpartum hemorrhage in our study as above mentioned. Because of that, the ACOG's new recommendations for definition of postpartum hemorrhage may be right, because none of our patients had symptoms of hemorrhage even if some patients hematocrit levels decreasing up to $10 \%$. Therefore, the SI values at 95 percentils occurred as 1 in our some patient. So that, further studies are needed to be discussed for new ACOG recommendation (11).

The strength of this study was designed prospectively and studied within a large population. The limitation of this study was that patients underwent CS were not included and patients with or without episiotomy/perineal tears were not evaluated separately because of the study design. In conclusion, $\mathrm{SI}$ can be used to assess the status of the 
mother and blood loss as a good parameter and should be noted in the patient folder for helping labor room workers who have some discrepancies among themselves to define $\mathrm{PPH}$. Of course, to diagnose a postpartum hemorrhage by using $\mathrm{SI}$, the reference values of the $\mathrm{SI}$ are needed first. In our study, reference values for shock indexes prepartum and postpartum 30. minutes, and 1st and 2nd hours after vaginal delivery were obtained while we used ACOG's recent recommendation for the definition of PPH as blood loss over $1000 \mathrm{ml}$ even in vaginal delivery.

Ethical Approval: The study was approved by the local ethics committee (Health Sciences University Gazi Yaşargil Training and Research Hospital clinical research ethics committee Date: 26/10/2018 protocol number: 166).

\section{Author Contributions:}

Concept: i.B.

Literature Review: i.B.,i.A.

Design : i.B.

Data acquisition: i.B.,i.A.

Analysis and interpretation I.B., I.A.

Writing manuscript: i.B.

Critical revision of manuscript: i.B.,i.A.

Conflict of Interest: The authors have no conflicts of interest to declare.

Financial Disclosure: Authors declared no financial support.

\section{References}

1. Allgöwer M, Burri C. Shock index. Dtsch Med Wochenschr. 1967 Oct 27;92(43):1947-50.

2. Rady MY, Nightingale $P$, Little RA, Edwards JD. Shock index: a re-evaluation in acute circulatory failure. Resuscitation. 1992 Jun-Jul;23(3):227-34.

3. Çakır E, Bindal A, Özçiftci Yılmaz P, Doğu C, Özkoçak Turan I. Sepsisli Hastaların Hemodinamik Destek ihtiyacı. Harran Üniversitesi Tıp Fakültesi Dergisi. 2020; 17(2): 172176.

4. Carlin A, Alfirevic Z. Physiological changes of pregnancy and monitoring. Best Pract Res Clin Obstet Gynaecol 2008;22(5):801-23.

5. Ouzounian JG, Elkayam U. Physiologic changes during normal pregnancy and delivery. Cardiol Clin 2012;30(3):31729. 288.

6. Tan EK, Tan EL. Alterations in physiology and anatomy during pregnancy. Best Pract Res Clin Obstet Gynaecol 2013;27(6):791-802. 290.

7. Birkhahn RH, Gaeta TJ, Bei R, Bove JJ. Shock index in the first trimester of pregnancy and its relationship to ruptured ectopic pregnancy. Acad Emerg Med. 2002;9(2):115119.

8. Peker N, Yavuz M, Aydın E, Ege S, Bademkıran M, Turan $G$, et al . Bilateral Hypogastric Artery Ligation: A tertiary center experience. Harran Üniversitesi Tıp Fakültesi Dergisi. 2019; 16(3): 526-529.

9. Say L, Chou D, Gemmill A, et al. Global causes of maternal death: a WHO systematic analysis. Lancet Glob Heal. 2014;2(6):e323-e333.

10. WHO Recommendations for the Prevention and Treatment of Postpartum Haemorrhage. Geneva: WHO Library; 2012.

11. American College of Obstetricians and Gynecologists.
Practice bulletin no. 183: postpartum hemorrhage. Obstet Gynecol 2017; 130:e168

12. Tunçalp Ö, Souza JP, Gülmezoglu M. New WHO recommendations on prevention and treatment of postpartum hemorrhage. Int J Gynecol Obstet. 2013;123(3):254 -256)

13. Borovac-Pinheiro A, Pacagnella $R$, Cecatti J et al. Postpartum hemorrhage: new insights for definition and diagnosis, Am J Obstet Gynecol. 2018 Aug;219(2):162-168

14. Ayadi AM El, Nathan HL, Seed PT, et al. Vital sign prediction of adverse maternal outcomes in women with hypovolemic shock: The role of shock index. PLoS One. 2016;11(2):1-12

15. Nathan H, El Ayadi AM, Hezelgrave N, et al. Shock index: an effective predictor of outcome in postpartum haemorrhage? BJOG An Int J Obstet Gynaecol. 2015 Jan 26;122(2):268 -75.

16. Le Bas A, Chandraharan E, Addei A, Arulkumaran S. Use of the "obstetric shock index" as an adjunct in identifying significant blood loss in patients with massive postpartum hemorrhage. Int J Gynecol Obstet. 2014;124(3):253 -255.)

17. American College of Obstetricians and Gynecologist Practice Bulletin: Clinical management guidelines for obstetrician-gynecologists. Postpartum hemorrhage. 2006. Obstet Gynecol 108:1039-47.

18. Gharoro EP, Enabudoso EJ. Relationship between visually estimated blood loss at delivery and postpartum change in haematocrit. J Obstet Gynaecol. 2009;29(6):517-520.

19. Pacagnella RC, Souza JP, Durocher J et al. A systematic review of the relationship between blood loss and clinical signs. PLoS One. 2013; 8: e57594.

20. Vandromme MJ, Griffin RL, Kerby JD, McGwin G Jr, Rue LW, Weinberg JA. Identifying risk for massive transfusion in the relatively normotensive patient: utility of the prehospital shock index. J Trauma. 2011 Feb;70(2):384-8

21. Borovac-Pinheiro A, Pacagnella RC, Morais SS, Cecatti JG. Standard reference values for the shock index during pregnancy. Int J Gynecol Obstet. 2016; 135: 11-15

22. Taylor D, Fleischer A, Meirowitz N, Rosen L. Shock index and vital-sign reference ranges during the immediate postpartum period. Int J Gynecol Obstet. 2017; 137: 192195

23. Borovac-Pinheiro A, Ribeiro FM, Morais SS, Pacagnella RC. Shock index and heart rate standard reference values in the immediate postpartum period: A cohort study PLoS One. 2019 Jun 11;14(6):e0217907.

24. Soma-Pillay P, Nelson-Piercy C, Tolppanen H, Mebazaa A. Physiological changes in pregnancy. Cardiovasc J Afr. 2016 Mar-Apr;27(2):89-94.

25. Papaioannou V, Chouvarda I, Gaertner E et al. Heart rate variability and cardiac baroreflex inhibition-derived index predicts pain perception in burn patients; PRONOBURN Group. Burns. 2016 Nov;42(7):1445-1454. doi: 10.1016/j.burns.2016.04.017

26. Nathan HL, Cottam K, Hezelgrave NL et al. Determination of Normal Ranges of Shock Index and Other Haemodynamic Variables in the Immediate Postpartum Period: A Cohort Study. Spracklen CN, editor. PLoS One. 2016; 11: e0168535

27. Tanacan A, Fadiloglu E, Unal C, Beksac MS. Importance of shock index in the evaluation of postpartum hemorrhage cases that necessitate blood transfusion. Women Health. 2020 Oct;60(9):1070-1078. 